\title{
Design and interpretation of non-inferiority studies: A clinician's perspective
}

\author{
Todd M. Brown, MD, MSPH ${ }^{\mathrm{a}}$ \\ a Division of Cardiovascular Disease, University of Alabama at Birmingham, Birmingham, AL
}

Received Jul 31, 2017; accepted Jul 31, 2017

doi:10.1007/s12350-017-1034-5

\section{INTRODUCTION}

The placebo-controlled, randomized clinical trial has long been considered the gold standard for establishing the efficacy of a new drug or treatment. ${ }^{1}$ Well validated statistical methods for the design, analysis, and interpretation of these studies exist, and clinicians are, in general, comfortable reading, interpreting, and applying data from these types of clinical trials into clinical practice. In these studies, investigators attempt to demonstrate the superior efficacy (superiority) of the new drug or treatment over placebo. However, frequently, there are scenarios which prohibit the use of a placebo-controlled, randomized clinical trial. In these situations, investigators are forced to compare the experimental therapy to an established treatment, referred to as an active comparison group, rather than placebo. Although investigators may choose to attempt to demonstrate superiority of the experimental treatment over the active comparison, in recent years, there has been an increase in the number of clinical trials that simply attempt to demonstrate the non-inferiority of this new therapy ${ }^{1-8}$ In this manuscript, I will summarize the role, design, and interpretation of this type of a clinical trial.

Electronic supplementary material The online version of this article (doi:10.1007/s12350-017-1034-5) contains supplementary material, which is available to authorized users.

The authors of this article have provided a PowerPoint file, available for download at SpringerLink, which summarises the contents of the paper and is free for re-use at meetings and presentations. Search for the article DOI on SpringerLink.com.

Reprint requests: Todd M. Brown, MD, MSPH, Division of Cardiovascular Disease, University of Alabama at Birmingham, LHRB 313, 1720 Second Avenue South, Birmingham, AL, 35294; tmbrown@uab.edu

J Nucl Cardiol 2017;24:1994-7.

$1071-3581 / \$ 34.00$

Copyright (c) 2017 American Society of Nuclear Cardiology.

\section{WHY PERFORM NON-INFERIORITY STUDIES?}

The ideal scenario for demonstrating the efficacy of a new drug or treatment would be to conduct a placebocontrolled, randomized clinical trial. However, in many cases, it would be unethical to do so. For instance, if there is a well-established treatment for a condition, it would be unethical to withhold that therapy simply to establish the efficacy of a new therapy over placebo. Furthermore, even if you were to demonstrate the efficacy of the new agent, one would still want to know how the new treatment compared to the established one. Thus, for both of these reasons, a head to head comparison of the new and established treatments, using the established therapy as an active control rather than a placebo control, is warranted. If the hypothesis is that the new treatment will have superior efficacy as compared to the established therapy, then the design of the clinical trial will be relatively straight forward. One would choose to conduct a randomized clinical trial with an active comparison rather than a placebo as the control group. All of the usual statistical methodology for the design and analysis of a traditional superiority trial could be utilized, simply substituting the active comparator in the place of the placebo as the control.

However, what if the new drug or treatment is unlikely to have superior efficacy when compared to the established therapy but has other advantages such as ease of administration, lower cost, or a better side effect profile? If one were to require that this new treatment demonstrate superior efficacy when compared to the established therapy, it might make it virtually impossible for this new treatment to succeed. The downside of that would be that a therapy that has roughly similar efficacy but additional advantages such as ease of administration, lower cost, or a better side effect profile would not be made available to patients. Thus, the concept of a noninferiority study design was introduced as a mechanism to establish that a new therapy had efficacy that was similar to, or at least not much worse than (non-inferior to), an established therapy. ${ }^{2-5}$ 


\section{DESIGN CONSIDERATIONS FOR NON- INFERIORITY STUDIES}

There are a number of design considerations that are unique to non-inferiority studies that need to be considered. The first, and possibly most important, is the establishment of the non-inferiority margin. ${ }^{1-8}$ The noninferiority margin is the degree to which the new therapy can be less efficacious than the established treatment and still be considered non-inferior. ${ }^{1-8}$ The reason for allowing some degree of decreased efficacy and yet consider the new treatment to be non-inferior is that the other advantages (ease of administration, lower cost, or a better side effect profile) would be so beneficial that it would be reasonable to give up a small amount of efficacy to obtain these other benefits. The challenge, however, is that there is no standard criteria to use to establish this non-inferiority margin. ${ }^{1-8}$

Take for example this hypothetical study as demonstrated in Table 1. Drug A was evaluated in a placebocontrolled, randomized clinical trial. The event rate in the placebo arm was $10 \%$. The event rate in the arm with Drug A was $5 \%$. This means that the absolute risk was $-5 \%$ (or an absolute risk reduction of 5\%). Then, let's say that the 95\% confidence interval around this absolute risk was $7 \%$ to $-3 \%$, demonstrating that Drug A was superior to placebo and would result in a 3-7\% reduction in absolute risk. This means that the smallest possible benefit of Drug A over placebo is a reduction in the absolute event rate of $3 \%$. Now, let's say that we conduct a non-inferiority study with a new agent, Drug B, and compare it to Drug A. In setting the non-inferiority margin, one needs to consider how much of that minimal $3 \%$ absolute risk reduction one is willing to give up to consider Drug B as non-inferior to Drug A. Some authors have suggested that maintenance of $50 \%$ of this treatment effect should be utilized to define the non-inferiority margin. ${ }^{1-8}$ In that scenario, Drug B would be considered non-inferior to Drug A as long as the upper bound of the confidence interval for the absolute event rate of Drug B as compared to Drug A was $\leq 1.5 \%$. In scenario 1 , Drug B has a $0.3 \%$ increased absolute risk as compared to Drug A with a $95 \%$ confidence interval of $-0.7 \%$ to $1.3 \%$. Because the upper bound of the confidence interval (1.3\%) is below the non-inferior margin threshold of $1.5 \%$, one would conclude that Drug B is non-inferior to Drug A. In scenario 2, Drug B has a $1 \%$ increased absolute risk as compared to Drug A with a 95\% confidence interval of $1 \%$ to $3 \%$. Because the upper bound of the confidence interval (3\%) is above the non-inferior margin threshold of $1.5 \%$, one would conclude that Drug B failed to demonstrate non-inferiority to Drug A.

The decision about how much efficacy to "give up" varies from study to study because it really depends on the situation at hand. ${ }^{1-8}$ For instance, if the efficacy outcome in question is mortality, one may not be willing to give up much efficacy. But, if the outcome is not as clinically important, one may tolerate a larger decrease in efficacy. ${ }^{1-8}$ Also, the magnitude of the benefit in terms of ease of administration, lower cost, or a better side effect profile needs to also be considered. If the side effect profile is something important like major bleeding, and the reduction in major bleeding is quite significant, one may be willing to trade slightly more efficacy than if the bleeding reduction were minimal. ${ }^{1-8}$ So, for all of these reasons, there are not clearly defined rules for how to establish the non-inferiority margin. However, one thing that all authors agree on is that the margin must be pre-specified by the investigators, and the rationale for how the margin was established needs to be clearly described. ${ }^{1-8}$

Beyond just setting the non-inferiority margin, one must also examine the clinical trial to see how well designed it was. Traditional superiority designed clinical trials benefit from the fact that poorly conducted clinical trials tend to bias the result towards the null hypothesis and thus make it less likely to prove the efficacy of a new therapy. ${ }^{2-5,8}$ However, in a non-inferiority design, one has to be very careful to examine the trial for design flaws that might bias the result towards the null as this may make it more likely to erroneously conclude that there is noninferiority. The population being studied should be as similar as possible to populations studied in previous clinical trials that established the efficacy of the established therapy that is being used as the control in the current study. ${ }^{2-5,8}$ In addition, the event rate in the established therapy arm should be similar to the event rate observed in earlier clinical trials. Only then, can the noninferiority of the new agent be established. ${ }^{2-5,8}$ If the established therapy performs poorly in the study either because of a poorly designed trial or because the study is done in a population with different characteristics, then the new therapy may only achieve non-inferiority due to poor study design rather than the merits of its efficacy.

The type of analysis must also be taken into account. Traditionally, clinical trials are evaluated on an intention to treat (ITT) principle. However, the ITT principle does have the tendency to bias the result towards the null hypothesis if there are a high number of cross overs or drop outs during follow-up or if trial participants do not adhere to therapies well. ${ }^{2-5}$ For all of these reasons, the ITT principle may not be the best method to analyze noninferiority studies. Given this, many authors have suggested that both an ITT analysis and a per-protocol (or as treated analysis), where only those who were adherent to the treatment assignments are analyzed, be performed and non-inferiority only be established if the non-inferiority margin is met under both types of analyses. ${ }^{2-5}$ 
Table 1. Hypothetical clinical trial scenarios of a Drug B compared to a Drug A in a non-inferiority clinical trial

$\begin{array}{lll}\text { Historical clinical trial } & & \\ \text { Placebo event rate } & \text { Drug A event rate } & \text { Absolute risk }(95 \% \mathrm{CI}) \\ 10 \% & 5 \% & -5 \%(-7 \%,-3 \%) \\ \text { Scenario } \mathbf{1} \text { (Drug B demonstrates non-inferiority } & \text { to Drug A) } & \\ \text { Drug A event rate } & \text { Drug B event rate } & \text { Absolute risk }(95 \% \mathrm{Cl}) \\ 5 \% & 5.3 \% & 0.3 \%(-0.7 \%, 1.3 \%) \\ \text { Scenario } \mathbf{2} \text { (Drug B fails to demonstrate non-inferiority }{ }^{\mathbf{a}} \text { to Drug A) } & \\ \text { Drug A event rate } & \text { Drug B event rate } & \text { Absolute risk }(95 \% \mathrm{Cl}) \\ 5 \% & 6 \% & 1 \%(-1 \%, 3 \%)\end{array}$

$C I$, Confidence interval

${ }^{a}$ Non-inferiority margin defined as $1.5 \%$ based on one-half of the absolute $3 \%$ reduction, which was the upper bound of the confidence interval for Drug A vs placebo in the historical clinical trial

\section{MISCONCEPTIONS ABOUT NON-INFERIORITY STUDIES}

There are some important misconceptions about non-inferiority studies that deserve mentioning. First, the failure to prove superiority in a traditionally designed, active comparison clinical trial is not the same as demonstrating non-inferiority. ${ }^{2,7}$ Given that there is no well-established method to determine the non-inferiority margin, it is very important that this margin be pre-specified and the criteria for how it was established well defined prior to conducting the study. It is considered inappropriate to define this margin after a therapy fails to demonstrate superiority in an active comparison clinical trial. ${ }^{2,7}$ The converse, however, is acceptable. If a drug or treatment not only demonstrates non-inferiority to an existing therapy but also superiority, it is acceptable to conclude that the new agent is indeed superior to the established therapy. ${ }^{2,7}$ This is because superiority is an objectively defined entity that is not subject to investigator bias.

Another misconception is that non-inferiority studies are done to minimize sample size. Remember, the effect size, or difference in treatment effect, between the two arms of a study affects the sample size needed. ${ }^{9}$ So, if the effect size is large, or one arm of the study is expected to have a much lower event rate than the other arm, then the sample size needed is lower. ${ }^{9}$ However, in the case of non-inferiority studies, the effect size is expected to be small by the nature of the non-inferiority design, as a larger effect size would justify a superiority design. ${ }^{4}$ As such, the sample size required is usually much larger than a traditional superiority design. ${ }^{4}$

Lastly, non-inferiority studies are not the same thing as equivalence trials. Equivalence trials seek to demonstrate that two agents are roughly similar in efficacy. ${ }^{1-8}$
However, in a non-inferiority study, one is not concerned about if the two agents are roughly similar but that the investigational agent is not much worse than the established agent. ${ }^{1-8}$ It could be much better than or even superior to the established agent.

\section{POTENTIAL CONCERNS WITH NON- INFERIORITY STUDIES}

Although non-inferiority studies have a role in evaluating new therapies, there are a few concerns with the interpretation of these studies that should be addressed. The first is assay sensitivity, which has already been discussed in part. Assay sensitivity is the ability of a study to distinguish effective therapies from those that are not effective. ${ }^{5,8}$ As mentioned earlier, many aspects of a non-inferiority study have the potential to bias it towards the null. In a traditional superiority designed clinical trial, this has the effect of making it more difficult to demonstrate the efficacy of an agent. However, in a non-inferiority study, it increases the changes that a new therapy is demonstrated to be non-inferior. ${ }^{5,8}$ Thus, more attention needs to be focused on the validity of the trial design and comparison with earlier studies while interpreting noninferiority analyses.

Second, there is significant concern for biocreep over time. ${ }^{5,8}$ Take for instance our previous example of Drug A and Drug B. Drug A was superior to placebo. Then Drug B was compared to Drug A, and was a little less efficacious than Drug A but non-inferior. Then, let's say Drug C is developed and compared to Drug B. Drug $\mathrm{C}$ might be slightly less efficacious than Drug B but noninferior to Drug B, and so on. The concern is that as you get down to Drug E, F, G, etc. you eventually end up with an agent that is no longer superior to placebo as 
each successive agent is a little less efficacious than the one that preceded it. ${ }^{5,8}$

\section{SUMMARY}

Non-inferiority studies are relatively new but becoming increasingly more popular. Frequently, new therapies are developed that have similar efficacy to established therapies but offer other advantages such as ease of administration, lower cost, or a better side effect profile. The non-inferiority study design allows investigators to demonstrate that a newer treatment is not less efficacious than an established therapy based upon a predetermined non-inferiority margin. Numerous concerns exist with the design and interpretation of these studies such as assay sensitivity and biocreep, but with careful design and analysis, these limitations can be overcome.

\section{Disclosures}

The author has no disclosures related to this work.

\section{References}

1. Gotzsche PC. Lessons from and cautions about noninferiority and equivalence randomized trials. JAMA 2006;295:1172-4.

2. Mulla SM, Scott IA, Jackevicius CA, You JJ, Guyatt GH. How to use a noninferiority trial. Users guide to the medical literature. JAMA 2012;308:2605-11.

3. Le Hananff A, Giraudeau B, Baron G, Ravaud P. Quality of reporting of noninferiority and equivalence randomized trials. JAMA 2006;295:1147-51.

4. Kaji Ah, Lewis RJ. Noninferiority trials. Is a new treatment almost as effective as another? JAMA 2015;313:2371-2.

5. Head SJ, Kaul S, Bogers AJ, Kappetein P. Non-inferiority study design: lessons to be learned from cardiovascular trials. Eur Heart J 2012;33:1318-24.

6. Piaggio G, Elbourne DR, Pocock SJ, Evans SJ, Altman DG, for the CONSORT group. Reporting of noninferiority and equivalence randomized trials. Extension of the CONSORT 2010 statement. JAMA 2012;308:2594-604

7. Schumi H, Wittes JT. Through the looking glass: Understanding non-inferiority. Trials 2011;12:106.

8. D'Agostino RB, Massaro JM, Sullivan LM. Non-inferiority trials: Design concepts and issues-The encounters of academic consultants in statistics. Stat Med 2003;22:169-86.

9. Brown TM. Power and sample size in clinical studies. J Nucl Cardiol 2015;22:1314-5. 